\title{
Current status and research progress of endoscopic retrograde cholangiopancreatography in the diagnosis and treatment of biliary and pancreatic system diseases
}

\author{
Li Yuehu ${ }^{1}$, Gong Jianp ${ }^{1}$, Shu Dong ${ }^{2 *}$
}

\author{
${ }^{1}$ Department of Hepatobiliary surgery, The second affiliated Hospital of Chongqing Medical University, Chongqing, \\ China \\ ${ }^{2}$ Department of General Surgery, Zhongxian Hospital of Traditional Chinese Medicine, Chongqing, China
}

Received: 28 June 2021

Accepted: 11 August 2021

\author{
*Correspondence: \\ Shu Dong, \\ E-mail: 1197159160@qq.com
}

Copyright: (c) the author(s), publisher and licensee Medip Academy. This is an open-access article distributed under the terms of the Creative Commons Attribution Non-Commercial License, which permits unrestricted non-commercial use, distribution, and reproduction in any medium, provided the original work is properly cited.

\begin{abstract}
Endoscopic retrograde cholangiopancreatography (ERCP) has been used clinically since the 1960s, after nearly half a century of development, it has become an important method for clinical diagnosis and treatment of biliary and pancreatic system diseases. In recent years, with the continuous updating of endoscopy equipment, the advancement of endoscopists' technology, and the progress in understanding of biliary and pancreatic diseases, the application of ERCP in the diagnosis and treatment of biliary and pancreatic diseases has become more extensive, and the diagnosis and treatment procedures have become more standardized. At the same time, the diagnostic role of ERCP has declined slightly with the advancement of noninvasive imaging technology. But, its intervention role in treatment is more prominent, reducing dependence on traditional trauma therapy. The aim of the study was to summarize the application status of ERCP technology in biliary and pancreatic system diseases and the research progress in recent years.
\end{abstract}

Keywords: Endoscopic retrograde cholangiopancreatography, Biliary tract diseases, Pancreatic diseases, Diagnosis, Therapy

\section{INTRODUCTION}

Diseases of the biliary and pancreatic system are common clinical diseases, and the diagnosis and treatment of diseases of the biliary and pancreatic system usually require inspection and manipulation of the bile duct or pancreatic duct. Traditional endoscopic retrograde cholangiopancreatography (ERCP) provides visualization of Vater ampulla (the entry point of the bile duct and pancreatic duct), and through the cooperation of contrast agent and X-rays, indirect visualization of the bile pancreatic duct is achieved. In recent years, with the continuous updating of flexible endoscopic instruments, the real visualization of the bile duct and pancreatic duct has been realized. At present, it has been widely used in the treatment of clinically difficult bile duct stones, the diagnosis and treatment of benign and malignant bile duct space occupations. And with the advancement of the endoscopist's technology and the advancement in understanding of biliary and pancreatic diseases, ERCP can be safely and effectively carried out in patients with changes in the normal anatomical structure of the gastrointestinal tract or bile pancreatic duct, elderly patients, children and pregnant women.

\section{DIAGNOSIS}

ERCP achieves the visualization of the ampulla and cholangiopancreatic duct through direct endoscopy and the use of contrast agents and interventions. So as to realize 
the diagnosis of biliary and pancreatic system diseases, with good sensitivity and specificity. With the development of imaging technology in recent years, the diagnostic role of ERCP in biliary and pancreatic diseases gradually declines. But in recent years, with the continuous updating of flexible endoscopic instruments, peroral cholangiopancreatoscopy (POCPS) has been widely used in the diagnosis of clinical benign and malignant biliary, and pancreatic lesions. And research shows that it makes up for the shortcomings of traditional ERCP 's low sensitivity. ${ }^{1}$

\section{Common bile duct stones}

ERCP is not recommended as a routine diagnosis of common bile duct stone due to its complications, liver function and abdominal ultrasound examination are the first choice for common bile duct stone. With the development of imaging, endoscopic ultrasound (EUS) and magnetic resonance cholangiopancreatography (MRCP) have been proven to have diagnostic sensitivity and specificity that are not weaker than ERCP in the diagnosis of common bile duct stone. And has gradually replaced ERCP in the diagnosis of common bile duct stones for the lower complication rate. EUS or MRCP is recommended to diagnose common bile duct stones in patients with persistent clinical suspicion but insufficient evidence of stones on abdominal ultrasonography. ${ }^{2}$

And research shows that EUS has higher sensitivity than MRCP, especially in the detection of small stones. There is no clear recommendation for the selection of MRCP and EUS. Depending on whether a history of gastrointestinal surgery, and cardiac pacemaker and other specific conditions appropriate inspection method selection. ${ }^{3}$ ERCP is currently only performed in patients with high suspicion of common bile duct stones, so that the treatment of common bile duct stones can be performed at the same time after diagnosis. ${ }^{4}$

\section{Pancreaticobiliary maljunction}

Pancreaticobiliary maljunction is usually caused by poor connection of the pancreaticobiliary duct. Because the action of the sphincter of Oddi does not regulate the function of the pancreaticobiliary junction in patients with pancreaticobiliary maljunction, two-way regurgitation occurs. Increases the risk of biliary malignancies and pancreatitis. ${ }^{5}$ Pancreaticobiliary maljunction is usually diagnosed with ERCP. With the development of imaging technology in recent years, multi-probe CT and MRCP have gradually replaced ERCP as the mainstream examination method due to their noninvasiveness. ${ }^{6}$

And because MRCP is not radioactive, it is often used as the first choice in pediatric patients. ${ }^{7}$ But in patients with relatively short common channel of pancreaticobiliary duct and complicated pancreaticobiliary duct connection, ERCP can directly prove whether the sphincter of Oddi plays a role in regulating the flow of bile and pancreatic juice. It has better diagnostic effect than CT and MRCP. ${ }^{8}$ However, it is usually performed when MRCP and multiprobe CT cannot make a clear diagnosis due to complications such as postoperative pancreatitis.

\section{Space-occupying lesions of the bile pancreatic duct and ampulla}

\section{Bile duct space occupying lesions}

For biliary space-occupying lesions of unclear nature, MRCP can be given priority to determine the condition of the biliary tract. For biliary strictures suspected of malignant lesions, ERCP can be performed, and previous studies have shown that the sensitivity and specificity of endoscopic ultrasound-guided fine-needle aspiration (EUS-FNA) in the diagnosis of biliary malignancies are slightly higher than that of ERCP. ${ }^{9}$ However, the combination of two endoscopic methods for sampling is better than the two methods alone in the diagnosis of suspected malignant bile duct stenosis. ${ }^{10}$ In recent years, with the advancement of flexible endoscopic instruments, the emergence of oral cholangiopancreatoscopy has made the bile duct directly visible.

And the use of controllable biopsy forceps (C-BF) increases the accuracy of tissue sampling, significantly improve the sensitivity and specificity of ERCP for diagnosing malignant biliary diseases. ${ }^{11,12}$ Studies have shown that ERCP combined with intraductal ultrasound (IDUS) is more sensitive and specific than EUS-FNA in diagnosing malignant lesions of the biliary tract. ${ }^{13}$ And biliary decompression therapy can be performed concurrently when necessary during the ERCP process.

\section{Pancreatic duct space occupying lesions}

For pancreatic duct occupying lesions, CT, MRI and EUS all have diagnostic sensitivity and specificity that are no less than or even better than ERCP. Therefore, ERCP is not recommended as a routine diagnostic test, CT is the first choice. Endoscopic ultrasound guided fine needle aspiration (EUS-FNA) also helps to obtain diseased tissue for biopsy. Identify focal autoimmune pancreatitis, pancreatic cysts and other benign lesions, and screen out precancerous lesions such as pancreatic intraepithelial neoplasia, intraductal papillary mucinous tumors, mucinous cystic tumors, etc.

And provide evidence for neoadjuvant chemotherapy before surgery for some pancreatic malignancies. ${ }^{14}$ Although EUS-FNA increases the probability of implantation and dissemination of malignant tumors, studies have shown that it is lower than the probability of dissemination caused by CT-guided percutaneous puncture.

In cases where CT, MRI, and EUS are not clearly diagnosed, further ERCP may be considered for cell 
brushing and tissue biopsy. Oral cholangiopancreatoscopy can provide higher sensitivity and specificity. ${ }^{11}$

\section{Ampullary space-occupying lesions}

ERCP is usually used to obtain tissue biopsy to clarify the nature of the placeholder when imaging examinations find space-occupying lesions in the ampulla. Research shows that biopsy of ampullary tissue does not increase the incidence of complications after ERCP. And ERCP has high diagnostic sensitivity and specificity. ${ }^{15}$ Visual inspection of the biliary and pancreatic ducts and intraductal ultrasonography can be performed during the operation, which helps to clarify the staging of malignant lesions of the ampulla, and can perform endoscopic treatment on the early malignant lesions of the ampulla at the same time.

\section{Oddi sphincter dysfunction (SOD)}

Oddi sphincter dysfunction usually considered to be patients who have other unexplained pancreaticobiliary pain after cholecystectomy, and it can cause pain by obstructing the flow of pancreas and bile. It can be divided into three types based on clinical manifestations: Type I refers to patients with elevated liver function and dilatation of the common bile duct; Type II includes one but not both of these criteria; Type III does not have the abovementioned manifestations. Currently, it is believed that ERCP pressure measurement is the only criterion for diagnosing SOD. ${ }^{16}$ At the same time as diagnosis, endoscopic sphincterotomy (EST) is feasible for treatment. Studies believe that EST is clearly effective for SOD type I, but the therapeutic effect of EST for SOD type II is still controversial, and SOD type III is not recommended for EST treatment. ${ }^{17}$

\section{TREATMENT}

ERCP can treat biliary and pancreatic system diseases such as bile duct stones, biliary tract strictures, biliary fistulas, and biliary malignancies through the use of various supporting equipment such as guide wires, arches, stents, balloons, and baskets under endoscopy. In recent years, with the development of POCPS, photodynamic therapy, and radiofrequency ablation of the biliary duct, the scope of application of ERCP in the treatment of biliary and pancreatic system diseases has gradually expanded.

\section{Common bile duct stones}

Common bile duct stones are often secondary to gallbladder stones, which can cause biliary obstruction, cholangitis, biliary pancreatitis, etc. For asymptomatic choledocholithiasis, the guidelines recommend early stone removal treatment, but recent studies have shown that ERCP stone removal for asymptomatic choledocholithiasis is not better than no treatment. Further research is needed to evaluate management strategies for asymptomatic choledocholithiasis. ${ }^{18}$
For patients with simple choledocholithiasis and choledocholithiasis combined with choledocholithiasis, there is no significant difference between ERCP and laparoscopic choledocholithotomy in the treatment effect and complications. ${ }^{19}$ However, ERCP is recommended for patients with a history of cholecystectomy. For patients with choledocholithiasis and gallbladder stones, ERCP and laparoscopic cholecystectomy can be performed at the same time. Studies have shown that it does not increase the probability of perioperative complications and reduces the length of hospital stay. ${ }^{20}$ For choledocholithiasis with larger diameter, it is feasible to combine papillary sphincterotomy with balloon dilation (ES-LBD), which has a good stone removal rate and safety. ${ }^{21}$ For patients with ES-LBD failure, laser lithotripsy can be performed with oral cholangiopancreatoscopy. Studies have shown that its safety and efficacy are not inferior to choledocholithotomy, and due to its minimal invasiveness, priority should be given. ${ }^{22}$ For patients with biliary pancreatitis, if they are accompanied by cholangitis and biliary obstruction, early ERCP is recommended, but early ERCP is not recommended for biliary pancreatitis without acute cholangitis. ${ }^{23}$

\section{Pancreatic duct stones}

Pancreatic duct stones are usually the sequelae of chronic pancreatitis, often accompanied by abdominal pain. Removing pancreatic duct stones to reduce the pressure on the bile duct can help relieve abdominal pain. For pancreatic duct stones with a smaller diameter (diameter $<5 \mathrm{~mm}$ ), simple ERCP has a higher success rate of stone retrieval. Lithotripsy is recommended for stones with larger diameters, and surgical treatment may be considered for pancreatic tail and multiple pancreatic duct stones. Extracorporeal shock wave lithotripsy (ESWL) has become a more mature lithotripsy method with a higher success rate and minimally invasive advantages. It can be repeated many times. It is recommended to decide whether to perform ERCP retrieving again based on the imaging results of the postoperative review. ${ }^{24}$ With the development of oral pancreatoscopy, electrohydraulic lithotripsy (EHL) and laser lithotripsy (LL) under pancreatoscopy have been widely used in the treatment of large diameter and difficult to remove pancreatic duct stones. And it has a success rate and safety that is not weaker than ESWL. LL has been proven to have a higher success rate than EHL. ${ }^{25}$

\section{Biliary fistula}

Biliary fistula is common in iatrogenic biliary tract injury caused by liver and biliary tract surgery, especially in laparoscopic cholecystectomy and partial hepatectomy, and can also be seen in traumatic liver and biliary tract injury. For patients with suspected biliary fistula, ERCP is not only a diagnostic method, but also a biliary stent and nasobiliary drainage at the same time. Studies have shown that the effective rate of ERCP for the treatment of postoperative biliary fistula can reach more than $88 \%$, which 
can reduce the rate of reoperation, and dilate the biliary tract injury. ${ }^{26}$

Endoscopic coil embolization of the bile duct and endoscopic injection of fibrin glue can be used as alternative treatments for refractory biliary fistulas that have failed endoscopic sphincterotomy, stent implantation and drainage. ${ }^{27,28}$

\section{BENIGN AND MALIGNANT BILIARY STRICTURES}

\section{Benign biliary stricture}

The most common causes of benign biliary stricture are postoperative injury and chronic inflammation. For biliary strictures diagnosed as benign lesions by long-term follow-up cytology and histological examination. ERCP is the first-line treatment for most patients, and the purpose of treatment is to achieve long-term biliary patency. Biliary stricture expansion and stent drainage are feasible under ERCP, and balloon dilatation for early postoperative stenosis $(<4$ weeks) can reduce the risk of biliary tract injury and biliary fistula. ${ }^{29}$ Balloon expansion combined with stent implantation is significantly better than simple balloon expansion in reducing the recurrence of biliary stricture. Indwelling multiple plastic stents is better than indwelling a single plastic stent, and the effect of using a fully covered metal stent is better than multiple plastic stents. ${ }^{30}$ For benign biliary strictures, bare metal stents are not recommended to avoid embedding the stent and cannot be removed under the endoscope. After stent implantation, follow-up is required and the stent is replaced in time to avoid cholangitis and stent blockage caused by long-term (>1 year) biliary stent implantation. ${ }^{31}$ Percutaneous transhepatic bile duct and EUS ultrasound guided puncture drainage and stent implantation are often used in patients who have failed ERCP and after gastrointestinal diversion surgery.

\section{Malignant biliary stricture}

Malignant biliary tract stenosis is common in biliary malignant tumors, pancreatic head tumors, duodenal papilla, etc. For malignant tumors that can be surgically removed, studies believe that preoperative biliary drainage has no obvious benefit for postoperative recovery and increases the incidence of complications. In addition to the symptoms of cholangitis and the patients who need to postpone the operation time due to neoadjuvant chemotherapy or other reasons, ERCP biliary drainage can be performed before the operation.

Full-membrane metal stent implantation can be used as the first choice. Routine ERCP biliary drainage is not recommended for other surgically resectable patients. For malignant biliary strictures that cannot be surgically removed, ERCP guided stent implantation, photodynamic therapy in the biliary tract, and radiofrequency ablation of the biliary tract are often used in the palliative treatment of malignant biliary strictures. ${ }^{32}$ For patients with symptoms of biliary obstruction, stent implantation under ERCP is the first choice for unobstructed drainage, and the first choice is a fully covered metal stent. Although the use of drug-eluting stents has shown potential efficacy in inhibiting tumor growth and prolonging survival, but there is still a lack of data support from clinical trials. ${ }^{33}$ ERCP guided photodynamic therapy and radiofrequency ablation of biliary tract have also been proven to delay tumor progression and relieve biliary obstruction. It is often used together with biliary stent implantation, which has a good effect on malignant tumors that grow in the biliary cavity. ${ }^{34,35}$ For patients who have failed ERCP treatment, EUS ultrasound guidance and percutaneous transhepatic bile duct drainage can be considered.

\section{ERCP FOR SPECIAL POPULATION}

With the advancement of endoscopists' technology and the advancement in understanding of biliary and pancreatic system diseases, ERCP can be safely and effectively carried out in patients with changes in the normal anatomical structure of the gastrointestinal tract or biliary and pancreatic ducts, elderly patients, children and pregnant women. It is safe and effective to implement ERCP in infants and children of different ages. It has a similar success rate and co-occurrence rate as adults, but it requires experienced endoscopists and children's matching models of ERCP equipment. ${ }^{36}$ It is safe and feasible to perform ERCP for elderly patients ( $>80$ years old), and the risk mainly comes from the patient's cardiopulmonary function and the choice of sedative drugs. ${ }^{37}$ Pregnant women can undergo ERCP biliary decompression when necessary to avoid adverse consequences to the pregnant woman and the fetus. ERCP can be carried out under lowdose radiation or no radiation with protective measures. Studies have shown that there is no consequence of fetal malformations. ${ }^{38}$ For patients with changes in the normal anatomy of the gastrointestinal tract or cholangiopancreatic duct, ERCP can also be performed through different approaches with the aid of balloon enteroscopy, laparoscopy or EUS. Balloon enteroscopy assisted ERCP is often the first choice due to its minimally invasive nature. ${ }^{39}$

\section{CONCLUSION}

With the development of imaging technology, the diagnostic effect of ERCP has gradually declined due to its complications. However, with the updating of endoscopic instruments and the understanding of biliary and pancreatic system diseases, the role of ERCP in interventional therapy has become increasingly prominent. Summarized as the following points: (1) for ERCP that is only diagnostic and pre-operative drainage of malignant biliary tract strictures, the indications should be strictly controlled, and the abuse of ERCP can be avoided to reduce the occurrence of complications; (2) with the emergence of oral cholangiopancreatoscopy, ERCP has a new application prospect in the diagnosis of 
cholangiopancreatic duct stenosis and the treatment of difficult cholangiopancreatic stones, which is worthy of further clinical research and promotion; (3) with the emergence of photodynamic therapy in the biliary tract and radiofrequency ablation in the biliary tract, new dawns have emerged in the treatment of unresectable malignant biliary tumors, and the therapeutic effects of drug-eluting stents still require a large amount of clinical data to support; (4) with the advancement of endoscopists' technology and the advancement in understanding of biliary and pancreatic system diseases, advanced age, children, pregnancy, and patients with changes in gastrointestinal anatomy are no longer contraindicated in ERCP. With the progress of future research, the application of ERCP in the diagnosis and treatment of biliary and pancreatic system diseases will definitely be further standardized and expanded.

Funding: No funding sources

Conflict of interest: None declared

Ethical approval: Not required

\section{REFERENCES}

1. Kurihara T, Yasuda I, Isayama H, Tsuyuguchi T, Yamaguchi T, Kawabe K, et al. Diagnostic and therapeutic single-operator cholangiopancreatoscopy in biliopancreatic diseases: Prospective multicenter study in Japan. World J Gastroenterol. 2016;22(5):1891-901.

2. Manes G, Paspatis G, Aabakken L, Anderloni A, Arvanitakis M, Soune P, et al. Endoscopic management of common bile duct stones: European Society of Gastrointestinal Endoscopy (ESGE) guideline. Endoscopy. 2019,51(5):472-91.

3. Yaghoobi MMME, Meeralam YM, Shammari KM. Diagnostic accuracy of EUS compared with MRCP in detecting choledocholithiasis: a meta-analysis of diagnostic test accuracy of head-to-head studies. Gastroint Endosc. 2017,86(6):986-93.

4. Williams E, Beckingham I, Sayed G, Gurusamy K, Sturgess R, Webster G, et al. Updated guideline on the management of common bile duct stones. Gut. 2017,66(5):765-82.

5. Kamisawa $\mathrm{T}$, Kaneko $\mathrm{K}$, Itoi $\mathrm{T}$, Ando $\mathrm{H}$. Pancreaticobiliary maljunction and congenital biliary dilatation. Lancet Gastroenterol Hepatol. 2017;2(8):610-8.

6. Ono A, Arizono S, Isoda H, Togashi K. Imaging of Pancreaticobiliary Maljunction. Radiographics. 2020;40(2):378-92.

7. Ishibashi H, Shimada M, Kamisawa T, Fujii H, Hamada Y, Kubota M, et al. Japanese clinical practice guidelines for congenital biliary dilatation. $\mathbf{J}$ Hepatobiliary Pancreat Sci. 2017;24(1):1-16.

8. Wang Q, Moon SB, Zang J, Liu J, Weng H, Wang X, et al. Usefulness of pre-operative endoscopic retrograde cholangiopancreatography in diagnosis and management of forme fruste choledochal cyst in children. ANZ J Surg. 2020;90(6):1041-5.
9. Moura DTH, Moura EGH, Bernardo WM, Moura ETH, Baraca FI, Kondo A, et al. Endoscopic retrograde cholangiopancreatography versus endoscopic ultrasound for tissue diagnosis of malignant biliary stricture: Systematic review and meta-analysis. Endosc Ultrasound. 2018;7(1):10-9.

10. Moura DTH, Ryou M, Moura EGH, Ribeiro IB, Bernardo WM, Thompson CC. Endoscopic Ultrasound-Guided Fine Needle Aspiration and Endoscopic Retrograde CholangiopancreatographyBased Tissue Sampling in Suspected Malignant Biliary Strictures: A Meta-Analysis of Same-Session Procedures. Clin Endosc. 2020;53(4):417-28.

11. Pereira P, Peixoto A, Andrade P, Macedo G. Peroral cholangiopancreatoscopy with the SpyGlass ${ }^{\circledR}$ system: what do we know 10 years later. J Gastrointestin Liver Dis. 2017;26(2):165-70.

12. Inoue $\mathrm{T}$, Kitano R, Kobayashi $\mathrm{Y}$, Ishii N, Sakamoto $\mathrm{K}$, Ohashi T, et al. Assessing the diagnostic yield of controllable biopsy-forceps for biliary strictures. Scand J Gastroenterol. 2018;53(5):598-603.

13. Inoue T, Kitano R, Kobayashi Y, Ishii N, Sakamoto $\mathrm{K}$, Ohashi T, et al. Assessing the diagnostic yield of controllable biopsy-forceps for biliary strictures. Scand J Gastroenterol. 2018;53(5):598-603.

14. Sun B, Hu B. The role of intraductal ultrasonography in pancreatobiliary diseases. Endosc Ultrasound. 2016;5(5):291-9.

15. Dacha S, Chawla S, Lee JE, Keilin SA, Cai Q, Willingham FF. Endoscopic retrograde cholangiopancreatography with ampullary biopsy vs ERCP alone: a matched-pairs controlled evaluation of outcomes and complications. Gastroenterol Rep. 2017;5(4):277-81.

16. Suarez AL, Pauls Q, Mauldin V, Cotton PB. Sphincter of Oddi Manometry: Reproducibility of Measurements and Effect of Sphincterotomy in the EPISOD Study. J Neurogastroenterol Motil. 2016;22(3):477-82.

17. Hyun JJ, Kozarek RA. Sphincter of Oddi dysfunction: sphincter of Oddi dysfunction or discordance? What is the state of the art in 2018 ? Curr Opin Gastroenterol. 2018;34(5):282-7.

18. Saito H, Kadono Y, Shono T, Kamikawa K, Urata A, Nasu J, et al. Remaining issues of recommended management in current guidelines for asymptomatic common bile duct stones. World J Gastroenterol. 2021;27(18):2131-40.

19. Zhu J, Li G, Du P, Zhou X, Xiao W, Li Y. Laparoscopic common bile duct exploration versus intraoperative endoscopic retrograde cholangiopancreatography in patients with gallbladder and common bile duct stones: a metaanalysis. Surg Endosc. 2021;35(3):997-1005.

20. Vettoretto N, Arezzo A, Famiglietti F, Cirocchi R, Moja L, Morino M. Laparoscopic-endoscopic rendezvous versus preoperative endoscopic sphincterotomy in people undergoing laparoscopic cholecystectomy for stones in the gallbladder and 
bile duct. Cochrane Database Syst Rev. 2018;4(4):10507.

21. Karsenti D, Coron E, Vanbiervliet G, Privat J, Kull E, Bichard P, et al. Complete endoscopic sphincterotomy with vs. without large-balloon dilation for the removal of large bile duct stones: randomized multicenter study. Endoscopy. 2017;49(10):968-76.

22. Li G, Pang Q, Zhai H, Zhang X, Dong Y, Li J, et al. SpyGlass-guided laser lithotripsy versus laparoscopic common bile duct exploration for large common bile duct stones: a non-inferiority trial. Surg Endosc. 2021;35(7):3723-31.

23. Lee HS, Chung MJ, Park JY, Bang S, Park SW, Song SY, et al. Urgent endoscopic retrograde cholangiopancreatography is not superior to early ERCP in acute biliary pancreatitis with biliary obstruction without cholangitis. PLoS One. 2018;13(2):190835.

24. Sharzehi K. Management of Pancreatic Duct Stones. Curr Gastroenterol Rep. 2019;21(11):63.

25. Saghir SM, Mashiana HS, Mohan BP, Dhindsa BS, Dhaliwal A, Chandan S, et al. Efficacy of pancreatoscopy for pancreatic duct stones: A systematic review and meta-analysis. World J Gastroenterol. 2020;26(34):5207-19.

26. Çolak S, Gurbulak B, Gok AFK, Çakar E, Bektaş H. Endoscopic treatment of postoperative biliary fistulas. Ulus Travma Acil Cerrahi Derg. 2020;26(1):103-8.

27. Maxia S, Prado L, Bozhychko M, Sanjuan C, Casellas JA, Aparicio JR. Endoscopic coil embolization of major intrahepatic biliary leak. Endoscopy. 2020;52(9):322-3.

28. Perisetti A, Raghavapuram S, Tharian B. Refractory cystic duct stump leak treated with fibrin glue. Endoscopy. 2019;51(7):170-1.

29. Hu B, Sun B, Cai Q, Wong LJY, Ma S, Itoi T, et al. Asia-Pacific consensus guidelines for endoscopic management of benign biliary strictures. Gastrointest Endosc. 2017;86(1):44-58.

30. Tomoda T, Kato H, Miyamoto K, Saragai Y, Mizukawa S, Yabe S, et al. Comparison Between Endoscopic Biliary Stenting Combined with Balloon Dilation and Balloon Dilation Alone for the Treatment of Benign Hepaticojejunostomy Anastomotic Stricture. J Gastrointest Surg. 2020;24(6):1352-8.
31. Sohn SH, Park JH, Kim KH, Kim TN. Complications and management of forgotten long-term biliary stents. World J Gastroenterol. 2017;23(4):622-8.

32. Dorrell R, Pawa S, Pawa R. Endoscopic Management of Malignant Biliary Stricture. Diagnostics. 2020;10(6):390.

33. Xiao JB, Weng JY, Hu YY, Deng GL, Wan XJ. Feasibility and efficacy evaluation of metallic biliary stents eluting gemcitabine and cisplatin for extrahepatic cholangiocarcinoma. World J Gastroenterol. 2020;26(31):4589-606.

34. Larghi A, Rimbaș M, Tringali A, Boskoski I, Rizzatti G, Costamagna G. Endoscopic radiofrequency biliary ablation treatment: A comprehensive review. Dig Endosc. 2019;31(3):245-55.

35. Moole H, Tathireddy H, Dharmapuri S, Moole V, Boddireddy R, Yedama $\mathrm{P}$, et al. Success of photodynamic therapy in palliating patients with nonresectable cholangiocarcinoma: A systematic review and meta-analysis. World J Gastroenterol. 2017;23(7):1278-88.

36. Keil R, Drabek J, Lochmannova J, Stovicek J, Koptova P, Wasserbauer M, Frybova B, et al. ERCP in infants, children, and adolescents-Different roles of the methods in different age groups. PLoS One. 2019;14(1):210805.

37. Yildırım AE, Ozturk ZA, Konduk BT, Balkan A, Edizer B, Gulsen MT, et al. The safety and efficacy of ERCP in octogenarians: a comparison of two geriatric age cohorts. Acta Gastroenterol Belg. 2017;80(2):263-70.

38. Azab M, Bharadwaj S, Jayaraj M, Hong AS, Solaimani P, Mubder M, et al. Safety of endoscopic retrograde cholangiopancreatography (ERCP) in pregnancy: A systematic review and meta-analysis. Saudi J Gastroenterol. 2019;25(6):341-54.

39. Yamauchi H, Kida M, Imaizumi H, Okuwaki K, Miyazawa $\mathrm{S}$, Iwai $\mathrm{T}$, et al. Innovations and techniques for balloon-enteroscope-assisted endoscopic retrograde cholangiopancreatography in patients with altered gastrointestinal anatomy. World J Gastroenterol. 2015;21(21):6460-9.

Cite this article as: Yuehu L, Jianp G, Dong S.

Current status and research progress of endoscopic retrograde cholangiopancreatography in the diagnosis and treatment of biliary and pancreatic system diseases. Int Surg J 2021;8:2866-71. 